\title{
LONGITUDINAL FVEP MEASUREMENTS IN PREMATURE NEONATES WITH POSTHAEMORHAGIC HYDROCEPHALUS: SENSITIVE MARKER FOR VENTRICULAR DILATATION?
}

\author{
Z. Rona ${ }^{1}$, C. Czaba ${ }^{1}$, M. Weninger ${ }^{1}$, A. Beke ${ }^{2}$, A. Pollak ${ }^{1}$, K. Klebermasz ${ }^{1}$ \\ ${ }^{1}$ Medical University Vienna, Vienna, Austria, ${ }^{2}$ Neonatology, Semmelweis University Budapest, I. Obs and \\ Gyn Clinic, Budapest, Hungary
}

Introduction: Intraventricular hemorrhage (IVH) is the most common cause of brain lesion in preterm infants. Among infants with IVH about 35-60\% develops posthemorrhagic hydrocephalus (PHH) which can lead to secondary injury. Flash Visual Evoked Potentials allow a noninvasive neurophysiological surveillance and may help defining the optimal time-point for intervention and prove its effectiveness with normalization of neurophysiological function.

Purpose of this study was to evaluate, if changes in fVEPs occur in preterm infants with progressive posthemorrhagic hydrocephalus before and after neurosurgical intervention.

Materials and methods: 17 patients admitted to the NICU at the Medical University of Vienna with progressive posthemorrhagic-hydrocephalus were evaluated by fVEP on a regular basis once weekly until therapeutic intervention (Shunt, EVD) for reducing intracranial pressure.

Results: In all 17 cases a change of VEP were found, wave latencies increased with ventricular dilatation and normalized mostly within a week of successful EVD implantation. There was a significant correlation $(\mathrm{r}=0,6 \mathrm{p}=0,013)$ between the severity of ventricle dilatation and the latency of the $\mathrm{N} 2$ wave. Elogation of the N2 wave latency with progressing hydrocephalus occurred before changes in cranial Doppler US, before clinical signs of elevated intracranial pressure and before the ventricular width exceeded $97^{\text {th }}$ percentile. There was also a significant correlation between the severity of the IVH and the pathological elongation of the $\mathrm{N} 2$ wave latency $(\mathrm{r}=0,5 \mathrm{p}=0,043)$.

Conclusion: VEP is of great value in the diagnostic and therapeutic management of preterm infants with progressive posthemorrhagic hydrocephalus and a sensitive marker for increasing intracranial pressure. 